\title{
NEW ARCHITECTURAL DESIGN OF A TEMPERATURE ROBUST MEMS GYROSCOPE WITH IMPROVED GAIN-BANDWIDTH CHARACTERISTICS
}

\author{
A.A. Trusov, A.R. Schofield, and A.M. Shkel \\ MicroSystems Laboratory, University of California, Irvine, CA, USA
}

\begin{abstract}
This paper reports a new MEMS vibratory rate gyroscope designed with increased robustness to fabrication imperfections and variations in environmental conditions. The distinct feature of the design is the increased response gain. The proposed architecture utilizes a single degree-of-freedom (DOF) drive-mode and a fully coupled 2-DOF sense-mode. The drive-mode operational frequency and the sense-mode bandwidth can be set independently, relaxing the tradeoff between the gain, die size, and detection capacitance, inherent to the previously reported robust gyroscopes with dynamic vibration absorber (DVA) architecture of the 2-DOF sense-mode. Prototypes with $2.5 \mathrm{kHz}$ operational frequency were characterized in air and demonstrated sense-mode $3 \mathrm{~dB}$ bandwidth of $250 \mathrm{~Hz}$. The uncompensated temperature coefficients of bias and scale factor were $313{ }^{\circ} / \mathrm{h} /{ }^{\circ} \mathrm{C}$ and $351 \mathrm{ppm} /{ }^{\circ} \mathrm{C}$, respectively. Using off-chip detection electronics, the rate sensitivity was 56 $\mu \mathrm{V} / \%$, ARW $-0.09 \% / \mathrm{s} / \sqrt{\mathrm{Hz}}$, bias instability $-0.08 \%$, and ARRW $-0.03 \%$ s $\sqrt{H z}$.
\end{abstract}

\section{INTRODUCTION}

The operation of micromachined vibratory gyroscopes is based on a transfer of energy between two modes of vibration caused by the Coriolis effect [1]. Conventional implementations often utilize single degree of freedom (DOF) drive- and sensemodes [2, 3]. In mode-matched implementations, the mechanical gain is proportional to the sense-mode quality factor, which allows increasing the sensitivity, theoretically unlimitedly. However, the increase in quality factor of a mode-matched gyroscope is always at the cost of sensor robustness, temperature drift, bandwidth, and limited linear operational range. Also, precise matching of the operational modes over temperature ranges defined by application requirements is practically challenging. Alternatively, the modes of operation can be designed with a certain frequency mismatch [2, 3]. Even though this approach improves the robustness and the bandwidth characteristics, the limitations of the design space (1DOF drive and 1-DOF sense) dictate a tradeoff between achieved robustness/bandwidth and gain.

For increasing robustness of vibratory gyroscopes, it is beneficial to design 1-DOF drive- and 2-DOF sense-modes so that the drive-mode resonant frequency is placed between the two resonant peaks of the sense-mode. A design of sense-mode with 2-DOF dynamic vibration absorber (VBA) architecture has been previously proposed and demonstrated to provide robust operation with a $200 \mathrm{~Hz}$ bandwidth using a micromachined prototype with a 750 $\mathrm{Hz}$ drive-mode operational frequency [4]. For this design concept, increasing the operational frequency would further increase the bandwidth, while also resulting in a decrease of the response gain. Adapting the DVA-based gyroscope design for operational frequencies above $1 \mathrm{kHz}$ while maintaining the sense-mode peaks at a practical spacing is challenging due to the limitation of the design space and involves a stringent tradeoff between the die size and detection capacitance [5].

Most real-world applications, such as automotive, military, and consumer electronics, require robust yet sensitive gyroscopes with operational frequencies above several $\mathrm{kHz}$ in order to suppress the effect of environmental vibrational noise [6]. At the same time, the desired mechanical bandwidth of the sense-mode is

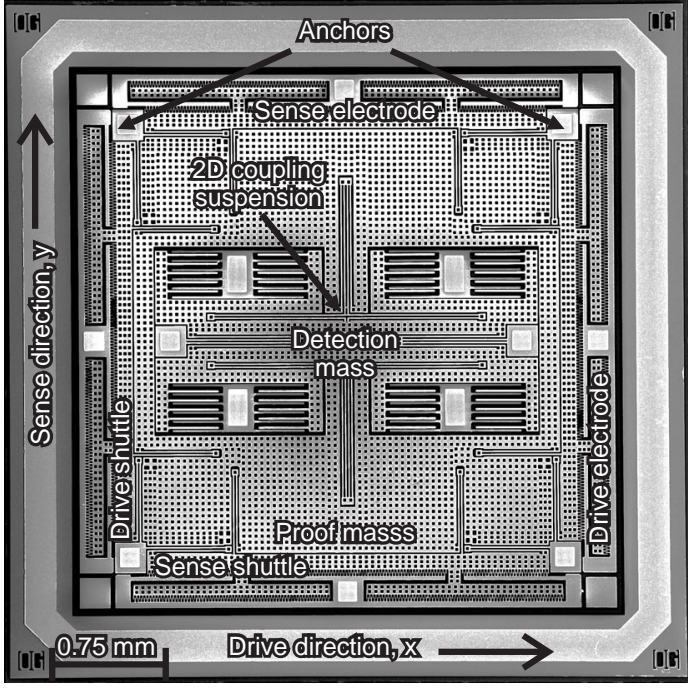

Figure 1: SEM image of a fabricated MEMS gyroscope with 1DOF drive-mode and a fully coupled 2-DOF sense-mode providing wide, temperature-robust bandwidth while minimizing the sacrifice in gain.

typically above $100 \mathrm{~Hz}$, but not more than $400 \mathrm{~Hz}$ [2, 3]. In this paper, we introduce an architecture, which extends the design space of the previously reported gyroscopes with 2-DOF sensemodes and overcomes the limitations imposed by the DVA dynamics. The device architecture is not just a minor optimization of previously reported designs, but rather a sharp conceptual deviation that introduces a new design architecture based on a different arrangement of structural components favorably shaping the response characteristics of robust gyroscopes.

\section{STRUCTURAL DESIGN AND DYNAMICS Structural Design}

The structural implementation of the proposed gyroscope is shown in Fig. 1. It implements the novel fully coupled architecture shown in Fig. 2. The device consists of an anchored outer frame, two drive-mode and two sense-mode shuttles, a proof mass, a detection mass, and a central anchor. Each of the two drive-mode and two sense-mode shuttles is suspended relative to the fixed frame by two springs. These flexures restrict the motion of the shuttles to their respective axes. Similar suspension elements couple the four shuttles to the proof mass. The described configuration of 4 shuttles, 16 suspension elements, and a proof mass forms a symmetrically decoupled suspension. This suspension architecture was introduced in [7] and later adapted by several groups [8, 9] for conventional vibratory gyroscopes with 1-DOF drive- and 1DOF sense-modes. In this work, the conceptual architecture of the suspension is utilized in a gyroscope with a 2-DOF sense-mode for the first time.

The proof mass $m_{p}$ is suspended in $x-y$ plane with total drive and sense axes stiffnesses of $k_{1}$. Using the electrodes on the drivemode shuttles, the mass is driven into a drive-mode oscillation to form a Coriolis element sensitive to rotation along the $\mathrm{z}$-axis. 
Frequency responses
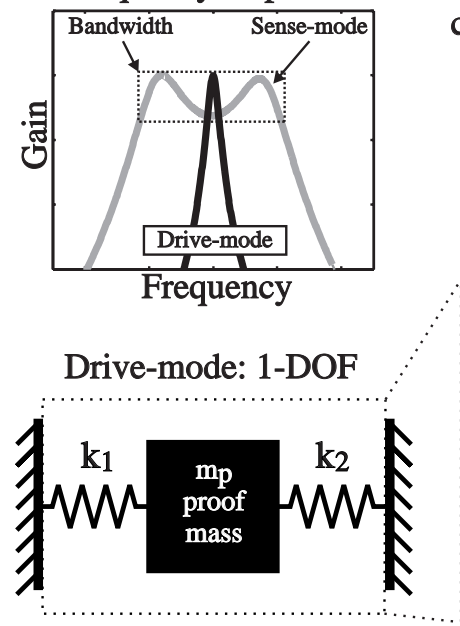

Sense-mode: coupled 2-DOF

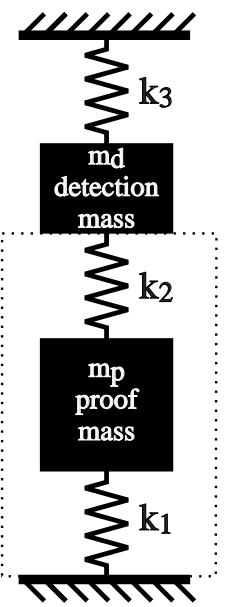

Figure 2: Drive- and sense-mode lumped models. The inset illustrates the response characteristics of the gyroscope.

Unlike the conventional case $[8,9]$, the Coriolis-induced motion is not directly picked-up from the proof mass, $\mathrm{m}_{\mathrm{p}}$, instead, the proof mass is coupled to a second, detection mass, $m_{d}$. The coupling flexure is bi-directional, with equal $\mathrm{x}$ and $\mathrm{y}$ stiffnesses $\mathrm{k}_{2}$. The detection mass is also coupled to the substrate with an inner suspension, $\mathrm{k}_{3}$. During rotation, the Coriolis acceleration of the proof mass is transferred to the detection mass, which responds in a wide frequency bandwidth due to the coupled dynamics of the proposed 2-DOF sense-mode.

Fig. 2 shows the lumped element drive and sense dynamic model of the proposed gyroscope. The drive-mode is a singleDOF system with mass $m_{p}$ and stiffness $k_{1}+k_{2}$. The sense-mode is a complete 2-DOF system, with two masses, $m_{p}$ and $m_{d}$, and three stiffnesses $\mathrm{k}_{1}, \mathrm{k}_{2}, \mathrm{k}_{3}$. The damping terms $\mathrm{c}_{1}, \mathrm{c}_{2}$, and $\mathrm{c}_{3}$ are located parallel to the respective spring elements.

\section{Dynamics and Selection of Parameters}

The drive-mode of the gyroscope is a single-DOF resonator with undamped natural frequency $\omega_{n}=\sqrt{ }\left(\left(k_{1}+k_{2}\right) / m_{p}\right)$. The sensemode is a 2-DOF dynamic system with in-phase and anti-phase resonant modes. If the y-axis displacement of the proof mass is considered as an output of the system, the corresponding transfer function has a zero ("anti-resonance" condition) at the frequency $\omega_{0}=\sqrt{ }\left(\left(k_{2}+k_{3}\right) / m_{d}\right)$. The proof mass anti-resonance is always located between the two sense-mode resonances and provides a convenient design guideline for selecting parameters of the system.

The available structural design parameters for the proposed gyroscope are the two masses $\mathrm{m}_{\mathrm{p}}$ and $\mathrm{m}_{\mathrm{d}}$ and three stiffnesses $\mathrm{k}_{1}$, $\mathrm{k}_{2}$, and $\mathrm{k}_{3}$. These five parameters define the location of the drivemode resonant frequency, i.e. the operational frequency of the gyroscope, and the locations of the two sense-mode resonant peaks, which define the bandwidth of the gyroscope. In practice, the operational frequency and bandwidth requirements are dictated by the specific application. We denote the desired operational frequency by $\Phi$ and the sense-mode peak spacing by $\Delta \Phi$. To ensure the optimal positioning of the drive-mode resonance with respect to the 2-DOF sense-mode response, we require that the drive-mode resonance coincides with the proof mass antiresonance in the sense-mode.

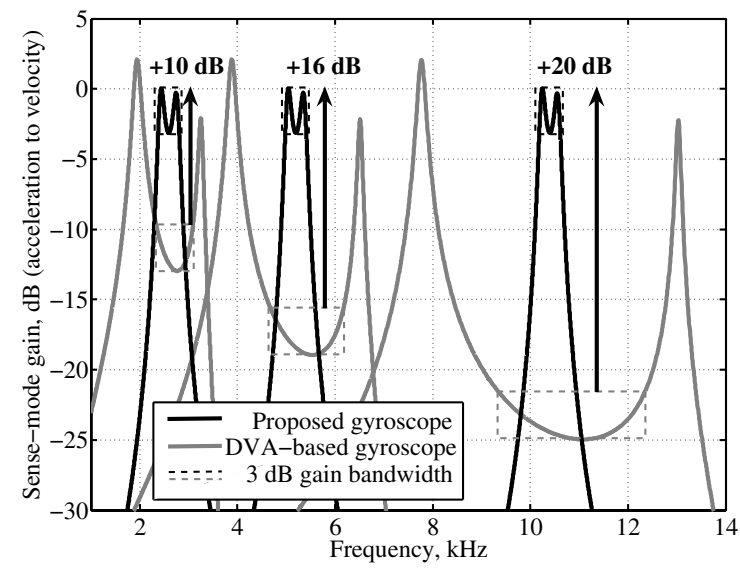

Figure 3: Frequency scaling of the sense-mode frequency response of the proposed and DVA-based gyroscopes.

We assume that the masses of the gyroscope together with the capacitive electrodes are implemented first. Then, the three stiffnesses $\mathrm{k}_{1}, \mathrm{k}_{2}$, and $\mathrm{k}_{3}$ become functions of the two masses $\mathrm{m}_{\mathrm{p}}$ and $\mathrm{m}_{\mathrm{d}}$, and selection of the desired operational frequency $\Phi$ and the frequency spacing $\Delta \Phi$. By solving the algebraic eigenvalue equation, the stiffnesses of the 2-DOF sense-mode are obtained,

$$
\left\{\begin{array}{l}
k_{1}=m_{p} \Phi^{2}-k_{2} \\
k_{2}=\Delta \Phi \sqrt{m_{p} m_{d}} \sqrt{\Phi^{2}-0.25 \Delta \Phi^{2}} \\
k_{3}=m_{d} \Phi^{2}-k_{2}
\end{array}\right.
$$

Unique solutions exist as long as $\Phi \geq \Delta \Phi / 2$, which holds for any physically meaningful combination of the operational frequency and the frequency spacing.

For both the proposed gyroscope and the DVA-based design [4], the gain is inversely proportional to the sense-mode peak spacing. In the DVA-based design, the peak spacing cannot be adjusted freely without a sacrifice in detection capacitance and/or enlargement of the die due to the $\mathrm{m}_{\mathrm{d}} / \mathrm{m}_{\mathrm{p}}$ mass ratio constraint [5]. This limitation is eliminated in the proposed device, where the peaks can be positioned arbitrary close to each other independent of the operational frequency and the mass ratio.

\section{Modeling of Gain and Bandwidth Scaling}

Fig. 3 shows effects of the operational frequency scaling on the frequency response of the sense-mode for both the proposed and the DVA-based gyroscopes. Based on the parameters of the experimentally characterized devices, we set the values of the proof and detection masses to $\mathrm{m}_{\mathrm{p}}=4.72 \mathrm{e}-7 \mathrm{~kg}$ and $\mathrm{m}_{\mathrm{d}}=1.35 \mathrm{e}-7 \mathrm{~kg}$. Assuming the device is operated in air, the damping coefficients are set to $c_{1}=1 \mathrm{e}-4 \mathrm{Ns} / \mathrm{m}, \mathrm{c}_{2}=5 \mathrm{e}-6 \mathrm{Ns} / \mathrm{m}$, and $\mathrm{c}_{3}=2 \mathrm{e}-4 \mathrm{Ns} / \mathrm{m}$. The operational frequency is iterated through $2.6,5.2$, and $10.4 \mathrm{kHz}$, while the desired sense-mode frequency spacing is kept constant at $350 \mathrm{~Hz}$. For the proposed gyroscope, the stiffnesses were obtained using (1).

The modeling confirms that the proposed design approach yields implementations with the prescribed operational frequency and the sense-mode frequency spacing is independent of the proof and detection mass values. Due to the flexibility of the extended design space of the proposed gyroscope, the two peaks can be placed close together. In this case, the sense-mode response of the detection mass has an increased bandwidth, composed of the two coupled resonant peaks and the region in between, while the gain is 


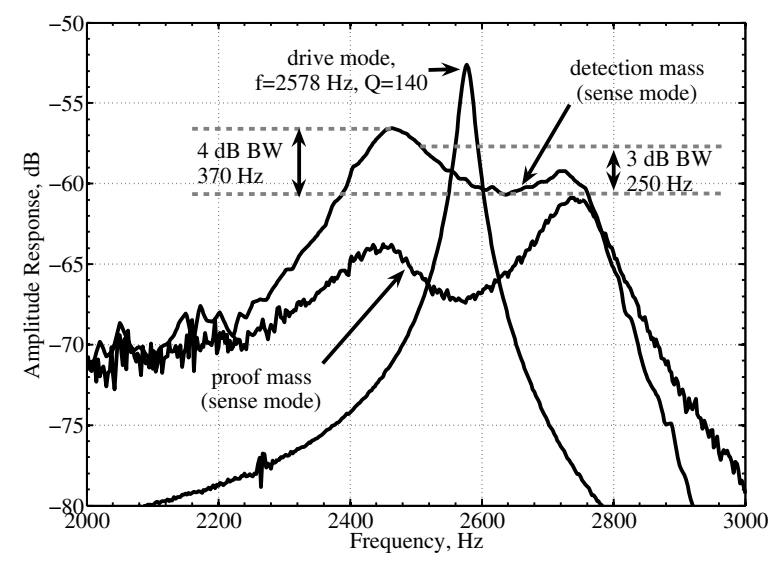

Figure 4: Experimental results demonstrate sense-mode bandwidth of several hundred $\mathrm{Hz}$; the drive-mode resonance is located between the sense-mode peaks.

comparable to the mode-matched case. The described configuration is preferable for applications requiring operational frequencies in $2-20 \mathrm{kHz}$ range and a bandwidth on the order of $100-350 \mathrm{~Hz}$.

\section{EXPERIMENTAL RESULTS}

\section{Fabrication}

The fabrication of prototypes was done using an in-house, wafer-level, two-mask process using SOI wafers with $50 \mu \mathrm{m}$ thick device layer and $5 \mu \mathrm{m}$ buried oxide layer. The first mask was used to define metallization of bonding pads using a lift-off process, while the second mask defined the structural layout. After patterning photoresist with the second mask, the wafers were subjected to a Deep Reactive Ion Etching (DRIE) using a Surface Technology Systems (STS) tool. Individual dies were released in a HF acid bath. An SEM image of a fabricated device with lateral-comb drive electrodes is shown in Fig. 1.

\section{Structural Characterization}

Experimental characterization of the lateral-comb device in atmospheric pressure is shown in Fig. 4. The measured drivemode resonant frequency was $2.58 \mathrm{kHz}$ which was located inbetween the 2-DOF sense-mode resonances at 2.47 and $2.73 \mathrm{kHz}$, and the drive-mode quality factor was 140 . A $250 \mathrm{~Hz} 3 \mathrm{~dB}$ bandwidth was formed in the sense-mode by the two resonant peaks and the flat region in-between.

\section{Thermal Characterization}

Temperature robustness of the gyroscopes, characterized by sensitivity of the bias and scale factor to temperature variations, is a critical performance parameter of gyroscopes targeted for realworld, temperature varying environments. The prototypes were experimentally characterized in variable temperature environment using a custom made, package-level heater equipped with a feedback control. Fig. 5(a) shows characterization of the temperature drifts of the single-DOF drive-mode operated in air. Increase in system temperature from $25{ }^{\circ} \mathrm{C}$ to $125{ }^{\circ} \mathrm{C}$ resulted in a $2.25 \mathrm{~dB}$ drop in gain, yielding a temperature coefficient of $2404 \mathrm{ppm} /{ }^{\circ} \mathrm{C}$. Temperature coefficients of this order are typical in conventional gyroscopes with 1-DOF sense-modes.

Drive-mode temperature drifts are easily mitigated by closed loop operation. However, the drop of gain in the sense-mode can be detrimental to accuracy of conventional mode-matched gyroscopes. In the proposed gyroscope, the temperature drift of the

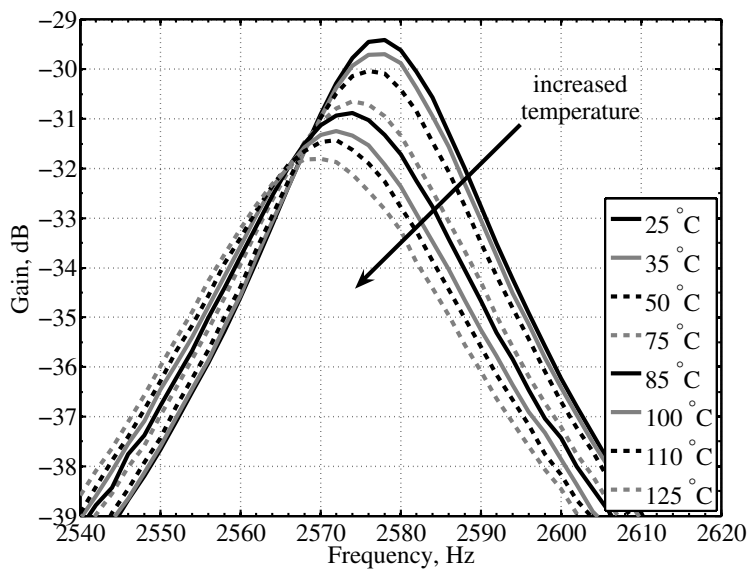

Figure 5(a): Effect of temperature variations on the drivemode in air, showing a gain temperature sensitivity of 2404 $\mathrm{ppm} /{ }^{\circ} \mathrm{C}$.

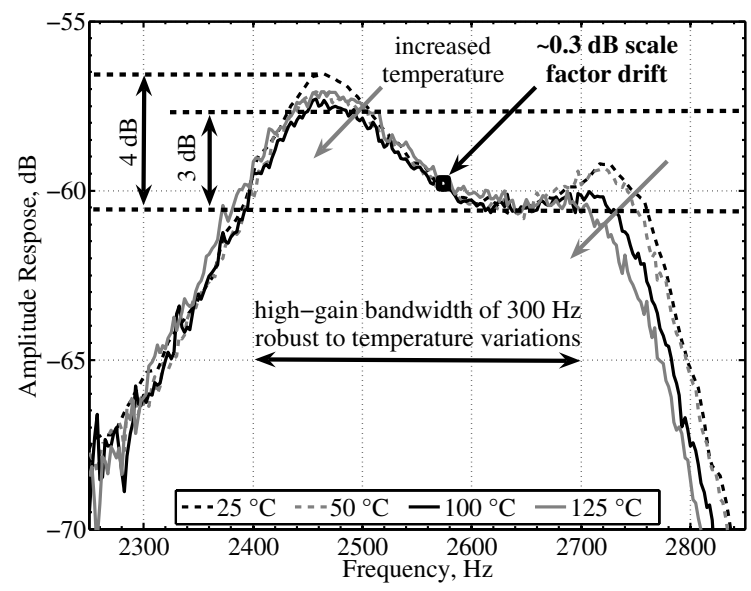

Figure 5(b): Effect of temperature variations on the sensemode in air, revealing a robust bandwidth of $300 \mathrm{~Hz}$. The uncompensated scale-factor temperature coefficient is approximately $351 \mathrm{ppm} /{ }^{\circ} \mathrm{C}$.

sense-mode is minimized by using the 2-DOF structure.

Sense-mode frequency-response of the detection mass was experimentally characterized at 4 different temperatures ranging from $25{ }^{\circ} \mathrm{C}$ to $125^{\circ} \mathrm{C}$, Fig. 5(b). Increase of temperature from 25 ${ }^{\circ} \mathrm{C}$ to $125^{\circ} \mathrm{C}$ results in approximately $1 \mathrm{~dB}$ change of the gain in a $300 \mathrm{~Hz}$ bandwidth. The temperature change in the sense-mode gain evaluated at the corresponding drive-mode resonance frequency is approximately $0.3 \mathrm{~dB}$ over a $100{ }^{\circ} \mathrm{C}$ range. This yields the scale-factor temperature coefficient of $351 \mathrm{ppm} /{ }^{\circ} \mathrm{C}$ - almost an 8 times improvement compared to the 1-DOF case of 2404 $\mathrm{ppm} /{ }^{\circ} \mathrm{C}$, measured using the $1-\mathrm{DOF}$ drive-mode. The temperature coefficient of the sense-mode phase was determined as $0.08 \%{ }^{\circ} \mathrm{C}$.

\section{Angular Rate Characterization}

The angular rate performance of the prototype was experimentally characterized in air using a computer controlled Ideal Aerosmith 1291BR rate table. The gyroscope was driven into 5 $\mu \mathrm{m}$ peak-to-peak resonant motion using a combination of a $30 \mathrm{~V}$ DC bias and a $3.5 \mathrm{Vrms}$ AC driving voltage applied to the anchored drive-mode lateral comb electrode. An electro-mechanical 


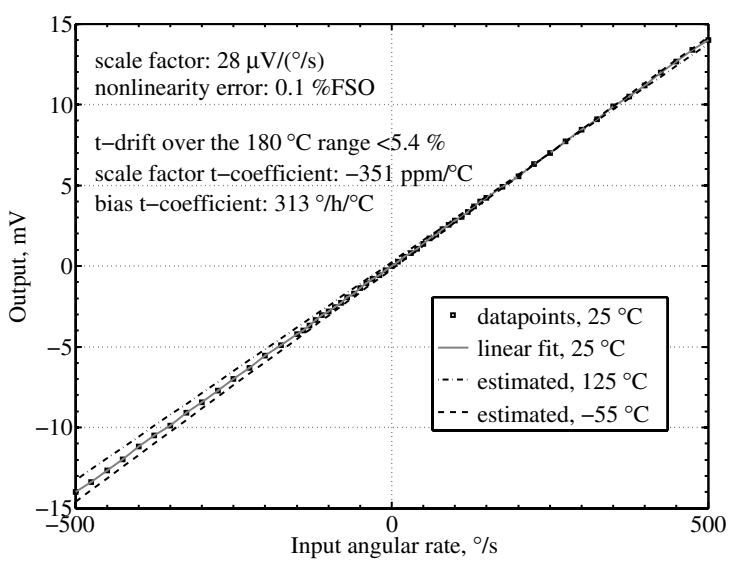

Figure 6: Experimental rate response at room temperature and estimated responses for $-55^{\circ} \mathrm{C}$ and $125^{\circ} \mathrm{C}$ temperatures.

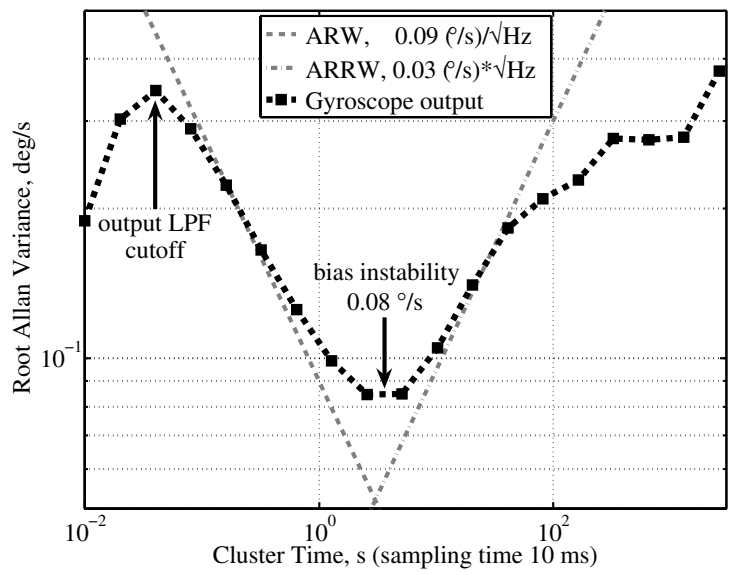

Figure 7: Allan Variance characterization of ARW, bias instability, and ARRW at zero rate, collected at $100 \mathrm{~Hz}$ for 4 hours.

amplitude modulation (EAM) technique was used to detect the Coriolis-induced motion in the sense-mode. The AC carrier voltage with $3.5 \mathrm{Vrms}$ amplitude at $20.5 \mathrm{kHz}$ frequency was applied to the mobile masses; the anchored sense-mode parallel-plate electrode was connected to the inverting input of an operational amplifier, configured as a transimpedance amplifier.

Fig. 6 shows the calibration curve obtained by programming the rate table to constant angular rate motion and observing the corresponding voltage output of the gyroscope. The collected datapoints were least squares fitted with a line to reveal the sensitivity of $28 \mu \mathrm{V} / \%$ s. As only a single sided capacitor was used, the total sensitivity of the device is $56 \mu \mathrm{V} / \%$ s for the same operational conditions. A $62.5 \%$ rate equivalent quadrature was measured by observing the out-of-phase output of the gyroscope at zero rate.

From the structural characterization results above, the gyroscope's scale factor temperature sensitivity was estimated as 351 $\mathrm{ppm} /{ }^{\circ} \mathrm{C}$. Based on the measured sense-mode phase temperature sensitivity and quadrature, the gyroscope's bias temperature sensitivity was estimated as $313 \% / \mathrm{h} /{ }^{\circ} \mathrm{C}$. Fig. 6 shows the rate response curves for $-55{ }^{\circ} \mathrm{C}$ and $125{ }^{\circ} \mathrm{C}$ temperatures calculated using the bias and scale factor temperature coefficients. The estimated maximum output drift over the $180{ }^{\circ} \mathrm{C}$ range is less than $5.4 \%$.

Fig. 7 shows experimental characterization of random noise modes using Allan Variance. The gyroscope's zero rate output was collected for 4 hours at $100 \mathrm{~Hz}$. With the off-chip detection electronics, the measured resolution (or angle random walk, ARW) at zero rate input was $0.09 \% / \mathrm{s} / \sqrt{\mathrm{Hz}}$, bias instability was $0.08 \% \mathrm{~s}$, and angle rate random walk (ARRW) was $0.03 \% \mathrm{~s} \sqrt{\mathrm{Hz}}$.

\section{CONCLUSIONS}

We presented a novel gyroscope design which utilizes a 1DOF drive-mode and a fully coupled 2-DOF sense-mode, comprising two masses with three suspension elements. To achieve optimal gain-bandwidth characteristics, the sense-mode bandwidth is defined by the two resonant peaks and the frequency region inbetween. Due to the high symmetry of the structure, the operational frequency is guarantied to be optimally placed between the sense-mode peaks even in presence of considerable fabrication imperfections. The rate sensitivity and quadrature of the gyroscope are comparable to the best reported performance numbers for MEMS gyroscopes operated in air [9]. At the same time, the gyroscope provides increased bandwidth as well as excellent robustness to fabrication imperfections and to in-operation temperature variations.

\section{ACKNOWLEDGEMENTS}

This work was supported by the National Science Foundation grant CMS-0409923, BEI Technologies contract BEI-36974, and UC Discovery program ELE04-10202. The authors would like to acknowledge Lynn E. Costlow and Cenk Acar of Custom Sensors $\&$ Technologies Systron Donner Automotive for the useful discussions and UCI Integrated Nanosystems Research Facility (INRF) for help with the fabrication of prototypes.

\section{REFERENCES}

[1] A. M. Shkel, "Type I and Type II Micromachined Vibratory Gyroscopes," Proceedings of the 2006 IEEE/ION PLANS, San Diego, CA, 4/25-27/06, IEEE/ION, (2006), pp. 586-593.

[2] M. Weinberg and A. Kourepenis, "Error Sources in In-Plane Silicon Tuning-Fork MEMS Gyroscopes," J Microelectromechanical Syst, 15, 3 (2006).

[3] N. Yazdi, F. Ayazi, and K. Najafi, "Micromachined Inertial Sensors," Proceedings of the IEEE, 86, 8 (1998).

[4] C. Acar and A. M. Shkel, "Inherently Robust Micromachined Gyroscopes with 2-DOF Sense-Mode Oscillator," J Microelectromechanical Syst, 15, 2 (2006).

[5] A. R. Schofield, A. A. Trusov, and A. M. Shkel, "Structural Design Tradeoffs for MEMS Vibratory Rate Gyroscopes with 2-DOF Sense Modes," Proceedings of the ASME 2007 IDETC \& CIE Conference, Las Vegas, NV, 9/4-7/07, ASME International, (2007).

[6] A. Madni, L. Costlow, and S. Knowles, "Common Design Techniques for BEI Gyrochip Quartz Rate Sensors for Both Automotive and Aerospace/Defense Markets," IEEE Sensors Journal, 3, 5 (2003).

[7] M. S. Kranz and G. K. Fedder, "Micromechanical Vibratory Rate Gyroscopes Fabricated in Conventional CMOS," Symposium Gyro Technology 1997, Stuttgart, Germany, 9/1617/97, (1997), pp. 3.0-3.8.

[8] W.-T. Sung, S. Sung, J. G. Lee, and T. Kang, "Design and Performance Test of a MEMS Vibratory Gyroscope with a Novel AGC Force Rebalance Control," J of Micromechanics and Microengineering, 17, 10 (2007).

[9] S. E. Alper, K. Azgin, and T. Akin, "A High-Performance Silicon-on-Insulator MEMS Gyroscope Operating at Atmospheric Pressure," Sensors and Actuators, A: Physical, 135, 1 (2006). 